\title{
Fundamental Limits for Implanted Antennas: Maximum Power Density Reaching Free Space
}

\author{
Anja K. Skrivervik, Marko Bosiljevac and Zvonimir Sipus
}

\begin{abstract}
Fundamental limits on antenna performances are of key interest to the antenna designer, as they allow fast assessment of the feasibility of specific antenna requirements. These limits are defined on key performance indicators (KPI) of specific antennas, as the directivity for large aperture antennas or the achievable bandwidth on electrically small antennas. These limits have been obtained considering that the antenna radiates into free space. In this contribution, we develop fundamental limits for implanted antennas, which thus radiate first into a lossy medium. Key performance indicators assessing the quality of a specific antenna radiating into lossy medium are the total radiated power reaching free space (out of the lossy host medium) and the maximum power density obtained at the surface of the lossy host medium. The fundamental limits for implanted antennas proposed in this paper yield upper bounds for both KPIs and have been obtained considering elementary sources radiating into a spherical phantom. Spherical wave expansion of the electromagnetic fields was used to determine all the fields, and the limits obtained yield a useful upper bound for more complex scenarios.
\end{abstract}

Index Terms-Fundamental limits, implantable antennas, spherical wave expansion

\section{INTRODUCTION}

$\mathrm{T}$ HEORETICAL and practical limits on antenna characteristics have been of huge interest for the system engineer since the beginning of wireless communications and radar systems. Examples start from the classic formula linking the far field limit to the antenna size, over closed form formulas giving the radiation characteristics for radiating apertures assuming the field distribution (see for instance [1]) to limits on side lobe levels in antenna arrays.

Limits on the performances of electrically small antennas have been studied since the early days of wireless transmissions. Indeed, contrary to electronic components, the antenna size for a given application is mainly determined by the laws of physics and is independent on technology: the antenna size with respect to the wavelength is the parameter

Manuscript submitted March 14, 2018., revised September 03, 2018., accepted December 18, 2018.

A. K. Skrivervik is with the Microwave and Antenna Group (MAG), Ecole Polytechnique Fédérale de Lausanne (EPFL), Lausanne CH-1015, (email:anja.skrivervik@epfl.ch).

M. Bosiljevac and Z. Sipus are with Faculty of Electrical Engineering and Computing, University of Zagreb, 10000 Zagreb, Croatia (e-mail: marko.bosiljevac@fer.hr, zvonimir.sipus@fer.hr).

All the authors contributed to the same extend to this manuscript. which will have the preponderant influence on the radiation characteristics.

The search of an explicit and if possible closed form relation between the electrical size of an antenna and its potential radiation performances has been a goal since the early radio days. Indeed, the HF and VHF frequencies used in those early days lead to large wavelengths and, in consequence, electrically small antennas. The pioneering work started by Chu [2], Wheeler [3], Harrington [4], and continued by Collin et al. [5], Fante [6], Fano [7] among others contains a large part of the theoretical developments and results which are still used by antenna engineers to assess the potential of an antenna.

The boom of mobile communication that started in the nineties with the mobile phone and continued with GPS, wireless sensors, and reached today with the wireless interconnection of virtually everything has rekindled the interest for electrically small antenna and fostered a new generation of work investigating the fundamental limitations of such radiating devices, aiming to refine the results achieved by the early pioneers [8-10], account for the antenna form factor [11-13] or account for losses inside the antenna [14, 15]. Finally, the seminal work of Gustafsson et al. [16-18] allowed obtaining the physical limitations on antennas of any shape by computing their static characteristics. It is interesting to notice that the key parameters for which limits where sought for were the quality factor of the antenna (linked to its bandwidth) and the antenna directivity. The aim of a good electrically small antenna design was thus to find the best compromise between volume, gain and bandwidth [19].

The next boom in wireless communications concerns wearable or implantable nodes with applications ranging from healthcare to sports over fashion tagging. These new wireless applications lead to the development of two new antenna families, the first for wearable wireless systems and the second for implantable ones. A review of wearable or implantable antennas can be found in [20-22], and recent attempts to design and characterize a capsule with antenna and wireless telemetry link can be found in [23] and [24].

The antennas for these body centered applications are in most cases electrically small antennas, as the frequency bands allotted to them lie mostly between $300 \mathrm{MHz}$ and $5 \mathrm{GHz}$, and the antenna size can be as small as few millimeters. However, the physical limitations cited above do not apply to these new antennas, as they were obtained considering that the antenna radiates into free space, or at least into a lossless media. 
Indeed most of the usual antenna theory assumes that the antenna radiates into free space, which is obviously not the case either for wearable or for implantable antennas. Radiation into a lossy medium has been considered in the past mainly in the frame of underwater communication [25], or the communication over a lossy ground [26]. In [25] it is clearly shown that fundamental antenna characteristics like the far field, the antenna radiation pattern or the bandwidth do not apply when the antenna radiates into an infinite lossy medium. Indeed, in the classic far field region of an antenna radiating into free space, the radiation intensity depends only on the angular coordinates $\theta$ and $\phi$, yielding the classic radiation patterns independent on the radial coordinate $r$. If the antenna radiates into a medium which is lossy, as the ocean for instance, the radiation intensity will also depend on the radial coordinate through the term $\exp (-2 \alpha r)$, and the resulting pattern will highly depend on the choice of the origin of the coordinate system. The case of the frequency bandwidth of an antenna radiating into a lossy medium defined as the band for which the latter's input reflection coefficient is below a certain value is also of limited interest since a low reflection coefficient does not necessarily signify that a large part of the power gets radiated, as it could also be dissipated into the host body.

In the case of implantable antennas, the lossy medium into which the antenna radiates is not infinite. It consists of the biological body hosting the wireless system, which is a complex inhomogeneous lossy medium of finite dimensions. The radiation properties of the system will thus not depend solely on the antenna, but also on the shape, dimensions and composition of the host body. Also, the near field coupling due to losses makes that the effect of the two cannot be separated, as the channel can be separated from the antenna in free space [27, 28]. The key radiation characteristic of an implantable antenna is thus the amount of power which reaches outside the body [29]. Some of the absorption in the host medium is unavoidable, as it is linked to the propagating wave traversing it before reaching free space. Another part, the losses due to the near field coupling to the biological medium is more difficult to assess, and to compare for different antennas.

In this paper we will first show how to differentiate between unavoidable propagation losses through the lossy medium, and avoidable losses due to near field coupling between the electromagnetic fields in the vicinity of the implanted antenna and the lossy medium hosting it. We will then propose some fundamental physical limits on the maximal power density that can reach free space for a specific antenna-biological host.

To this aim, we will consider the canonical case of elementary electromagnetic sources placed in a spherical body phantom. The latter can consist of several concentric layers to model the typical biological body layers (e.g. skin, muscle, bone); the accuracy of spherical phantoms used for characterization of implanted antennas is discussed in [30]. To analyze the properties of this canonical case, we use a numerical tool based on spherical waves expansion which has been presented earlier $[31,32]$ and has the advantage of being computationally very fast. From the results obtained, we can first understand the absorption mechanism in the lossy body and enhance the model in order to provide an upper bound for the power density reaching outside the host body. The paper is organized as follows: Section II presents the spherical wave model used, which was already partly described in [31] but which is required to understand the following sections. Section III presents results obtained with the spherical wave model proposed in section II, while section IV presents the derivation of a physical limit yielding the maximum power density reaching outside the lossy body as a function of the distance of the implant to the body surface and the dimension of the implant.

\section{SPHERICAL BODY MODEL}

A spherical model of a human body provides worthwhile and useful results despite being only a rough approximation $[4,30]$. Therefore, the analyzed structure, shown in Fig. 1, is composed of a sphere (with radius $r_{\text {body }}$ ) and of an implanted antenna. The sphere modeling the body can be either homogeneous or formed by concentric layers in order to mimic a part of the human body (skin, fat, muscle, bone, for instance) by using dielectric properties that are similar to those of real human tissues (values are taken from [30, 33]). Since we are interested in the basic properties of the implanted antenna, it is modeled as a small sphere with radius $r_{\text {impl }}$ (filled with air) and a current source (either an electric or a magnetic dipole). The implanted antenna itself is located at a distance $r_{\text {feed }}$ from the center of the body, and both parallel and perpendicular orientations of the antenna (defined by the orientation of the current source vector relative to the nearest outer interface of the sphere) are investigated.

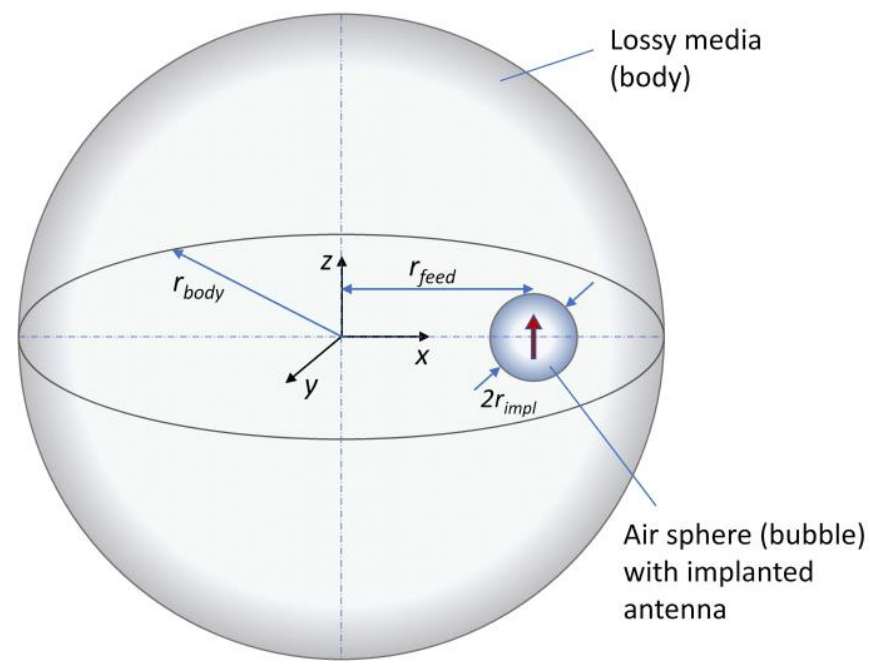

Fig 1. View of the analyzed structure with the excitation moved away from the center.

The solution procedure makes use of the spherical-wave modal expansion. The electromagnetic field in a spherical structure (with zero free-charge density) can be represented 
using vector spherical harmonics [34,35] as:

$$
\begin{aligned}
& \mathbf{E}=-\sum_{n} \sum_{m} a_{m n} \mathbf{M}_{m n}+b_{m n} \mathbf{N}_{m n}, \\
& \mathbf{H}=-\frac{j}{\eta} \sum_{n} \sum_{m} b_{m n} \mathbf{M}_{m n}+a_{m n} \mathbf{N}_{m n} .
\end{aligned}
$$

where

$$
\begin{gathered}
\mathbf{M}_{m n}=\nabla \times \mathbf{r} \psi_{m n}, \quad \mathbf{N}_{m n}=\frac{1}{\beta} \nabla \times \mathbf{M}_{m n} \\
\psi_{m n}(\beta, r, \theta, \phi)=\frac{1}{\beta r} \hat{Z}_{n}(\beta r) P_{n}^{m}(\cos \theta) e^{j m \phi}
\end{gathered}
$$

Here $\psi_{m n}$ is the elementary solution of the Helmholtz differential equation, i.e. $\hat{Z}_{n}$ denotes Schelkunoff type of spherical Bessel or Hankel functions [35], and $\beta$ denotes the wavenumber of the considered media. For the outgoing waves the electromagnetic field components can be explicitly written as (e.g. the E-field components):

$$
\begin{aligned}
\mathbf{E}= & -\frac{1}{\beta r} \sum_{n=0}^{\infty} \sum_{|m| \leq n} a_{m n} e^{j m \phi} \hat{H}_{n}^{(2)}(\beta r) \\
& \cdot\left(\hat{\theta} \frac{j m}{\sin \theta} P_{n}^{|m|}(\cos \theta)-\hat{\phi} \frac{d}{d \theta} P_{n}^{|m|}(\cos \theta)\right) \\
& +b_{m n} e^{j m \phi}\left(\hat{r} \frac{n(n+1)}{\beta r} \hat{H}_{n}^{(2)}(\beta r)+\hat{H}_{n}^{\prime(2)}(\beta r)\right. \\
& \left.\cdot\left(\hat{\theta} \frac{d}{d \theta} P_{n}^{|m|}(\cos \theta)+\hat{\phi} \frac{j m}{\sin \theta} P_{n}^{|m|}(\cos \theta)\right)\right)
\end{aligned}
$$

We can also define the mode impedance for the outgoing spherical modes. For the electric sources the mode impedance is equal

$$
Z_{m n}^{J}=\frac{E_{\theta}}{H_{\phi}}=-\frac{E_{\phi}}{H_{\theta}}=j \eta \frac{\hat{H}_{n}^{(2)}(\beta r)}{\hat{H}_{n}^{(2)}(\beta r)},
$$

while for magnetic type of sources we can define:

$$
Z_{m n}^{M}=\frac{E_{\theta}}{H_{\phi}}=-\frac{E_{\phi}}{H_{\theta}}=-j \eta \cdot \frac{\hat{H}_{n}^{(2)}(\beta r)}{\hat{H}_{n}^{(2)}(\beta r)}
$$

Here $\eta$ represents the wave impedance of the considered media. Note that the mode impedance does not depend on the $\phi$-index $m$. It is only a function of the radial coordinate and of the order of the Hankel function (radial index $n$ ).

As can be seen in Fig. 1 the structure of interest consists of two spherical structures - the spherical model of a body and the spherical model of an implanted antenna. Each spherical structure can be multilayered and can be separately analyzed using the spherical-wave modal expansion approach described with eqs. (1)-(3) (if the structure is multilayered it can be analyzed using a reflection and transmission matrix approach, a vector-Legendre approach or an equivalent circuit analysis approach, see [36-38] for details). Therefore, the main challenge in the analysis of the structure in Fig. 1 lies in connecting two spherical problems that have displaced centers of coordinate systems.

If we denote spherical harmonics in the global coordinate system (related to spherical body) with $\mathbf{M}_{m n}(\beta, r, n, m)$ and $\mathbf{N}_{m n}(\beta, r, n, m)$, and in the local coordinate system (related to the implanted antenna) with $\mathbf{M}_{\mu \nu}^{\prime}\left(\beta, r^{\prime}, v, \mu\right)$ and $\mathbf{N}_{\mu \nu}^{\prime}\left(\beta, r^{\prime}, \nu, \mu\right)$, then these two representations can be connected using addition theorems, see [39-41] for details.

The EM fields in the implanted antenna sphere and in the outer sphere (human body) are "matched" using the equivalence theorem. In more details, using Love's equivalence principle we have defined two equivalent problems: (a) the equivalent implanted antenna problem consisting of an implanted antenna surrounded with air, and (b) the equivalent spherical body problem in which the implanted antenna is replaced with a dielectric of permittivity equal to the one of implanted antenna surrounding material. The spherical harmonics representations of two equivalent problems should fulfill the boundary condition that the tangential EM-field is continuous at the boundary of the small sphere (containing the implanted antenna).

The boundary between the body and the surrounding free space, as well as the multilayer body case, is included into the outside equivalent problem using the scattered field approach. The scattered field from the outer boundary and from the rest of the multilayer spherical structure $\tilde{E}_{\text {scat }}$ and $\tilde{H}_{\text {scat }}$ is calculated by transforming the EM-fields from the local to the global coordinate system using the following scheme (here ' $\sim$ ' denotes one component of the spherical-wave modal expansion given with eq. (1)):

$$
\begin{aligned}
& \left\{\begin{array}{c}
\tilde{J}_{e q}\left(r_{i m p l}^{\prime}, v, \mu\right) \\
\tilde{M}_{e q}\left(r_{i m p l}^{\prime}, v, \mu\right)
\end{array}\right\} \Rightarrow\left\{\begin{array}{c}
\tilde{E}_{e q}\left(r^{\prime}, v, \mu\right) \\
\tilde{H}_{e q}\left(r^{\prime}, v, \mu\right)
\end{array}\right\} \\
& \Rightarrow\left\{\begin{array}{c}
\sum_{n, m} \tilde{E}_{e q}(r, n, m) \\
\sum_{n, m} \tilde{H}_{e q}(r, n, m)
\end{array}\right\} \Rightarrow\left\{\begin{array}{c}
\sum_{n, m} \tilde{J}_{e q}\left(r_{e q}, n, m\right) \\
\sum_{n, m}^{n} \tilde{M}_{e q}\left(r_{e q}, n, m\right)
\end{array}\right\} \\
& \Rightarrow\left\{\begin{array}{c}
\sum_{n, m} \tilde{E}_{s c a t}(r, n, m) \\
\sum_{n, m} \tilde{H}_{s c a t}(r, n, m)
\end{array}\right\} \Rightarrow\left\{\begin{array}{c}
\sum_{v, \mu} \tilde{E}_{s c a t}\left(r_{i m p l}^{\prime}, v, \mu\right) \\
\sum_{v, \mu} \tilde{H}_{s c a t}\left(r_{i m p l}^{\prime}, v, \mu\right)
\end{array}\right\}
\end{aligned}
$$

Here $r_{\text {eq }}$ represents the radius of the sphere defined in the global coordinate system containing equivalent currents that radiate the same EM field as the equivalent currents defined in the local coordinate system at $r^{\prime}=r^{\prime}$ impl (both related to the outside equivalent problem). In other words, in order to calculate the scattered field from the outer body boundary (and from other layers in the multilayer structure) one needs to 
transform the excitation from local to global coordinate system. Furthermore, in order to match the fields at the boundary of a small sphere one needs to transform the scattered field back from the global to local coordinate system.

Details about the implemented analysis method can be found in [31].

\section{FIRST RESULTS AND FINDINGS}

The aim of this section is to understand the propagation in a lossy medium and the different mechanisms contributing to the losses, by analyzing some simple canonical scenarios of elementary sources placed in a spherical phantom using the described spherical wave decomposition method. To this aim, we will first recall some of the results already published in [31], before deepening our understanding from different points of view. The geometry considered, if not otherwise specified, is the one used in [31] and described in Fig. 1, with a working frequency of $403.5 \mathrm{MHz}$, a phantom sphere $r_{b o d y}=9 \mathrm{~cm}$ radius and permittivity $\varepsilon_{\mathrm{r}}=43.50-j 34.75$ [33, IEEE Head model].

In [31], we showed that as expected as the losses in the phantom are of an electric type, the total power reaching outside the body was higher for a magnetic source than for an electric dipole. What was less intuitive was that for the considered phantom having the dimension of about one wavelength in the tissue, the total power reaching free space almost did not depend on the position of the source. When the electric dimension of the phantom increased (considering higher frequencies for instance), the position started to have some effect. What was also shown was that the level of the electric field just outside the phantom showed a lens effect due to the body. This focusing effect is strongly dependent on the position of the source inside the body. Finally, it was shown in [31] that the radius of the air bubble containing the source had a crucial importance on the amount of total power reaching free space; as smaller the bubble is, the larger is the near field coupling between the dipole and the lossy phantom.

Fig. 2 shows the total radiated power in the phantom reaching out of a sphere of radius $R$ for an electric and a magnetic source placed at the centre of the phantom, considering an air bubble of radius $r_{\text {impl }}=0.1 \mathrm{~cm}$ around the source. Note that the total radiated power from the body is given with respect to $1 \mathrm{~mW}$ input power emitted by the current source (i.e. entering the lossy medium); it can be calculated directly from the spherical mode expansion using Parseval's theorem [36]. We can separate three zones in the phantom: the first, where the reactive near-field absorption dominates, the second, characterized by an exponential decay due to propagating field absorption, and the third at the discontinuity at the phantom-air boundary. Note that the reactive near-field region is partly in the air sphere containing the implanted antenna, and partly in the lossy body.

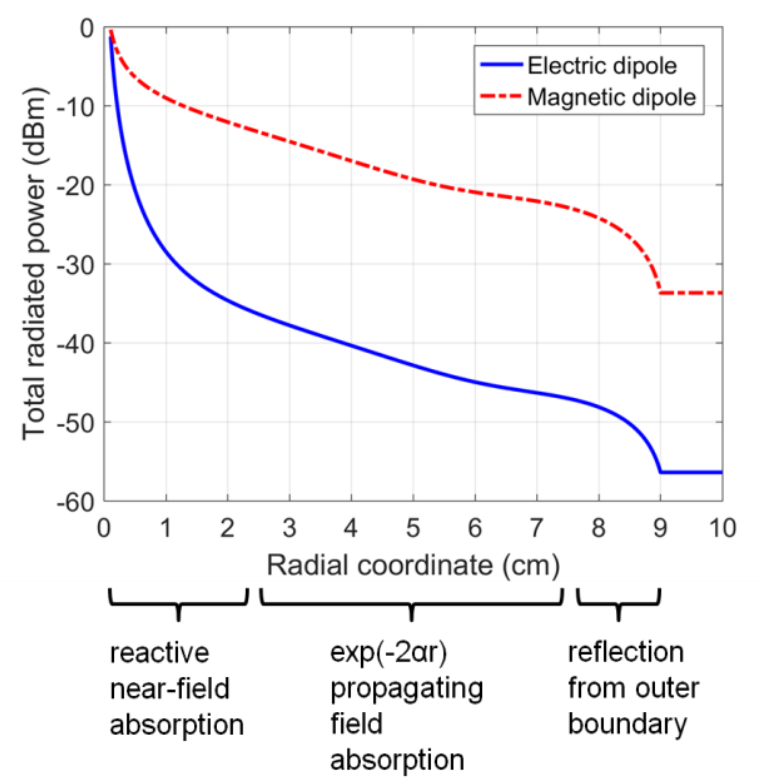

Fig. 2. Total radiated power in the phantom reaching out of a sphere of radius $R$ (radial coordinate) for an electric and a magnetic source placed at the centre of the phantom at $403 \mathrm{MHz}, r_{\mathrm{impl}}=0.1 \mathrm{~cm}$.

In order to investigate a bit more on the crucial contribution of the near field coupling to the losses, Fig. 3 represents the total radiated power reaching free space as a function of the radius of the air bubble, $\mathrm{r}_{\text {impl }}$, for different position of the source inside the phantom. We see clearly that the total radiated power increases with the radius of the air bubble, which stems from the decrease of the near field losses. We also see that the benefits of increasing the lossless area around the sources reach saturation after a certain radius, as we reach the limit of the reactive near field. Furthermore, for air bubble large enough, there is practically no difference between electric and magnetic source since the reactive near-field is concentrated in the bubble and thus the main differentiation effect is not present in a lossy media (body). Finally, as indicated earlier, the position of the bubble has very little effect in this scenario.

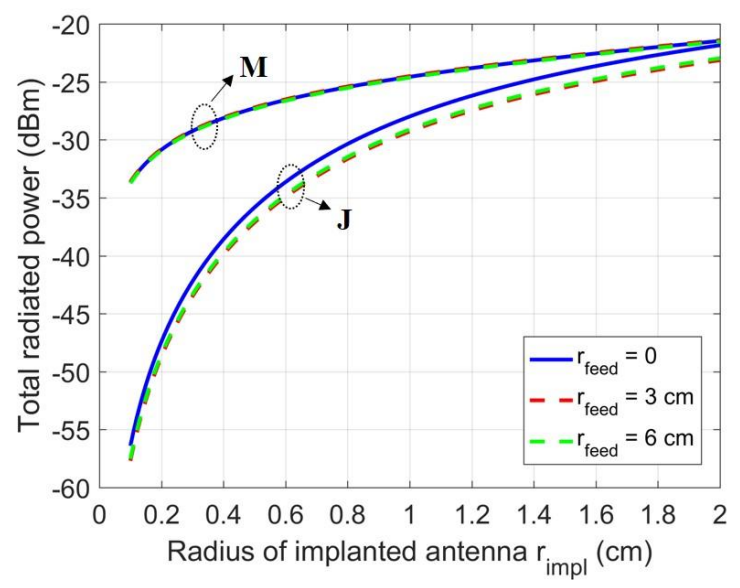

Fig, 3 : Total radiated power reaching free space as a function of the radius of the air sphere encapsulating the electric source $\left(r_{\text {impl }}\right)$, for different positions of the latter. The source is parallel to the phantom-air interface when it approaches the latter. 
The effect of increasing the bubble is shown from a different perspective in Fig. 4, where the total power reaching out of a sphere of radius $R$ inside the phantom is shown for an electric and a magnetic source placed at the centre, but for different radii of the lossless air bubble surrounding the sources.

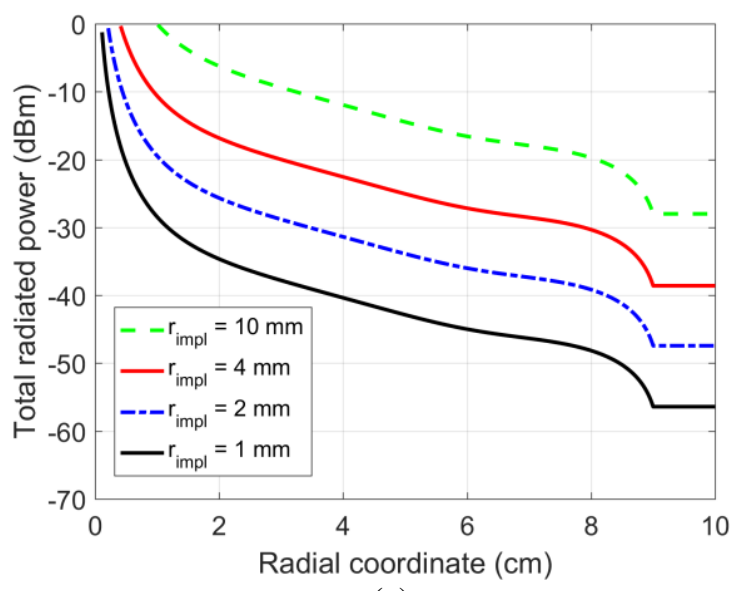

(a)

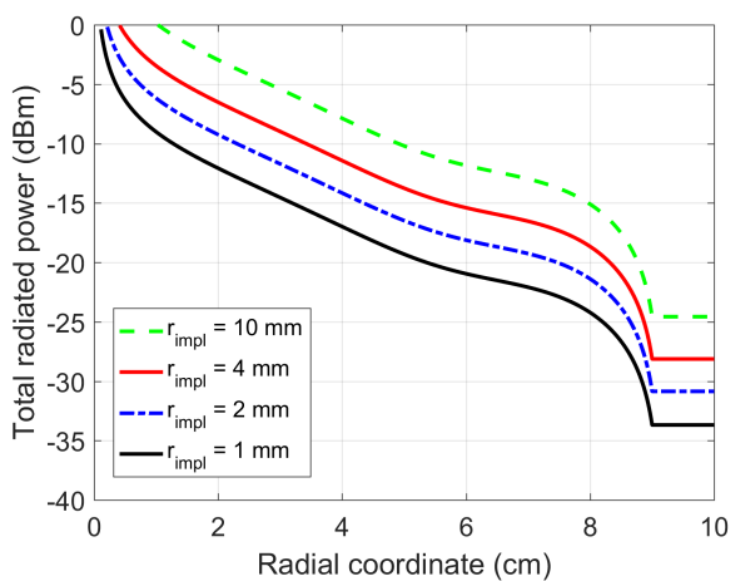

(b)

Fig. 4. Total radiated power in the phantom reaching out of a sphere of radius $\mathrm{R}$ (radial coordinate) for a source placed at the centre of the phantom at 403 $\mathrm{MHz}, r_{\text {impl }}=0.1,0.2,0.4$ and $1 \mathrm{~cm}$; (a) electric source, (b) magnetic source.

The three different areas in the phantom (reactive near field, propagating field absorption and edge discontinuity) are again clearly seen, but we see that the reactive near-field area in the lossy media (body) becomes smaller when the size of the lossless encapsulations increases (since the outer radius of the reactive near-field region is not changed).

The presented results also hold for other frequencies and permittivities. However, it is hard to make a general conclusion how the power density and the total radiated power behave depending on frequency and (complex) permittivity, in particular since the permittivity of biological tissues vary a lot with frequency. Furthermore, we cannot change the permittivity of biological tissues, i.e. we need "to live with permittivities we have". Generally speaking, larger tissue conductivity (i.e. larger imaginary part of permittivity) will cause larger losses related to all three effects - reactive near field losses, propagating field losses and losses due to reflection. A larger magnitude of permittivity (with the same ratio between real and imaginary part of permittivity) will decrease the radius of the reactive near field region and, therefore, the reactive near field losses will be smaller; however, the other two effects will be larger. A change of frequency will have a similar effect (since the wave number is proportional to the frequency), except for the reflection coefficient losses which in principle will not change unless the phantom is small in terms of wavelength (there is a strong reactive near-field effect outside a small body which will cause that the reflection losses are enlarged if the frequency is reduced).

The following conclusions can be drawn from these results:

- The exponential absorption of signal as it travels through the lossy phantom is unavoidable and has to be lived with.

- A large amount of the losses is due to the reactive near field. These losses can be avoided, by using the lossless encapsulation around the source.

- Finally, if the total radiated power reaching out of the phantom does not depend much on the position of the source in the latter, the EM field just outside the body depends highly on this position, due to the lens effect of the body [31].

In the case of an implanted antenna, it seems thus that power density usable for a link with a transceiver located out of the body will depend mainly on four factors:

1. The dielectric and conductive characteristics of the body at the considered frequency

2. The type of the antenna (electric, magnetic or a combination of both)

3. The distance to the body-free space interface

4. The diameter of the lossless encapsulation of the antenna.

In the next section, we will develop a rigorous closed form formula giving the upper limit of this power density, according to the frequency, the phantom characteristics, the distance from the antenna to the interface and the radius of its lossless encapsulation.

\section{THEORETICAL RESULTS AND LiMITS}

The excitation used so far was the dominant spherical mode only (i.e. the lowest spherical mode radiated by electric or magnetic point source) since the losses of higher order modes are much higher and they do not contribute significantly in the overall results. This is visible in Fig. 5. where a comparison between dominant mode excitation and higher modes excitation for both, electric and magnetic type sources is shown. We can see that the radius of the near-field reactive zone for higher order modes is much larger and leads to a faster decay of radiated power. 


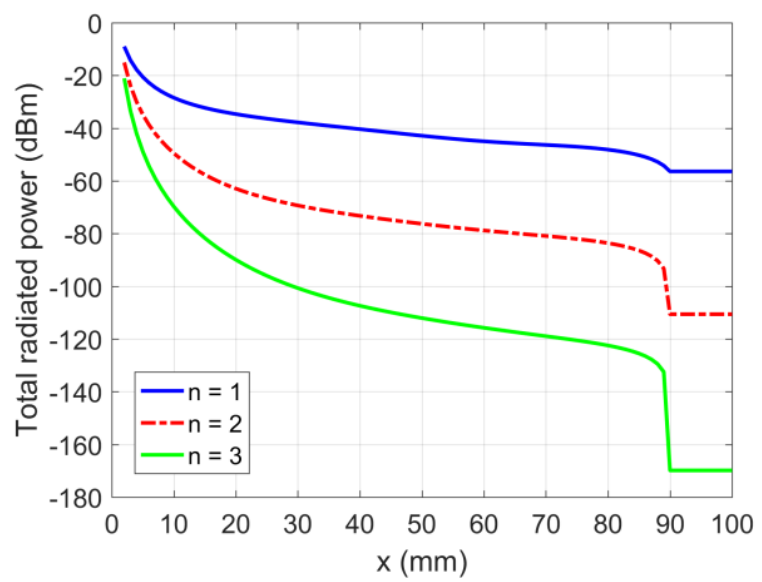

(a)

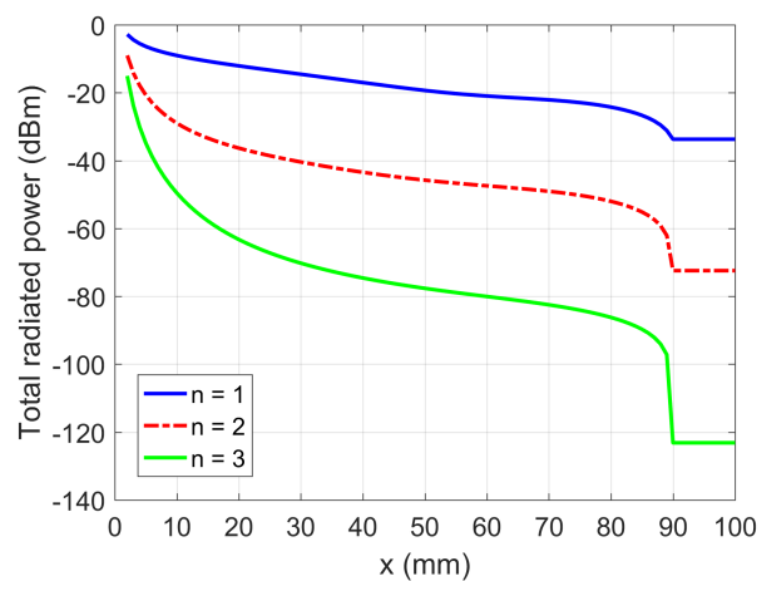

(b)

Figure 5. Total radiated power evaluated for specific modes along different radii away from the source, (a) electric dipole case, (b) magnetic dipole case.

To clearly identify the problem with higher-order modes Fig. 6. shows the spherical mode impedance for the considered cases. We notice that the wave impedance is predominately reactive when $|\beta r|<n$ (the imaginary part prevails) and almost constant when $|\beta r|>n$ (here the real part prevails, see [4]). In other words, in the volume $|\beta r|<n$ the EM field is predominately reactive or non-radiating, i.e. most of the EM energy is circulating around the implanted antenna and, consequently, is absorbed in the tissue. Furthermore, radius at which the reactive fields stop to dominate depends on the order of the excited spherical mode (and on the permittivity of the considered media).

Therefore, the goal is to excite the spherical modes with index $n$ as small as possible, i.e. the goal is to excite the spherical modes with $n=1$.

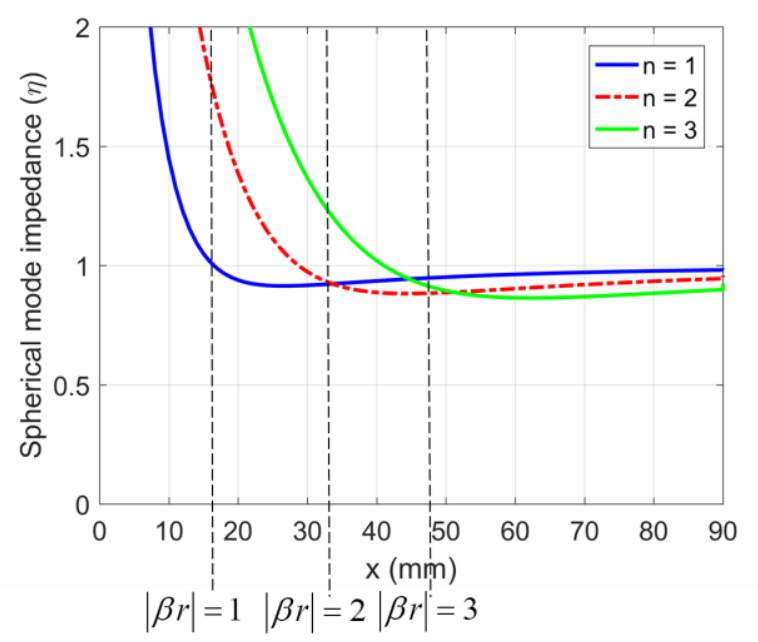

(a)

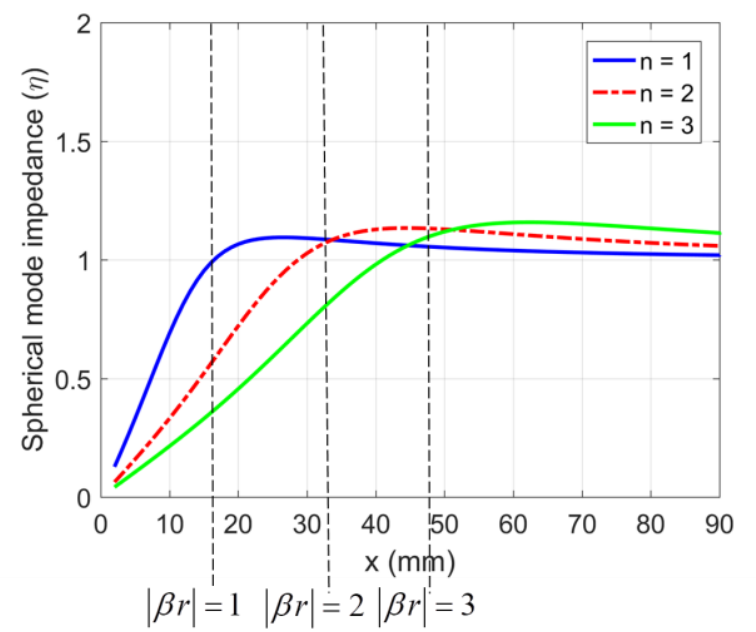

(b)

Figure 6. Magnitude of spherical mode impedance evaluated for specific spherical modes along different radii away from the source, (a) electric dipole case, (b) magnetic dipole case.

Once focused on the dominant mode we can express the power density for this case (electric dipole excitation in lossless media, see eqs. (1)-(4))

$$
\begin{aligned}
W_{r} & =E_{\theta} \cdot H_{\phi}^{*} \\
& =\frac{j}{\eta}\left|b_{01}\right|^{2} \frac{1}{(\beta r)^{2}}\left(\frac{d}{d \theta} P_{1}(\cos \theta)\right)^{2} \hat{H}_{1}^{(2)}(\beta r)\left(\hat{H}_{1}^{(2)}(\beta r)\right)^{*} \\
W_{\theta} & =-E_{r} \cdot H_{\phi}^{*} \\
& =-\frac{j}{\eta}\left|b_{01}\right|^{2} \frac{2}{(\beta r)^{3}} P_{1}(\cos \theta)\left(\frac{d}{d \theta} P_{1}(\cos \theta)\right)\left|\hat{H}_{1}^{(2)}(\beta r)\right|^{2}
\end{aligned}
$$

or, more specifically [42]:

$$
W_{r}=E_{\theta} \cdot H_{\phi}^{*}=C^{2} \frac{\sin ^{2} \theta}{r^{2}} \cdot \eta \cdot\left(\beta^{2}-j \frac{1}{\beta r^{3}}\right)
$$




$$
W_{\theta}=-E_{r} \cdot H_{\phi}^{*}=C^{2} \frac{2 \cos \theta \sin \theta}{r^{2}} \cdot \eta \cdot\left(j \frac{\beta}{r}+j \frac{1}{\beta r^{3}}\right)
$$

Here the constant $\mathrm{C}$ is equal $C=\left|b_{01} / \eta \beta^{2}\right|$. If the propagation constant is replaced with a complex propagation constant $\bar{\beta}=\left(2 \pi / \lambda_{0}\right) \sqrt{\varepsilon_{r}}=\beta-j \alpha$ since we are in the lossy homogeneous media, the expressions become

$$
\begin{aligned}
& W_{r}=E_{\theta} \cdot H_{\phi}^{*}= C^{2} \frac{\sin ^{2} \theta}{r^{2}} \cdot \bar{\eta} \\
& \cdot\left\{|\beta|^{2}+\frac{2 \alpha}{r}+\left(1-\frac{\bar{\beta}^{*}}{\bar{\beta}}\right) \frac{1}{r^{2}}-j \frac{1}{\bar{\beta} r^{3}}\right\} \cdot e^{-2 \alpha r} \\
& W_{\theta}=-E_{r} \cdot H_{\phi}^{*}=C^{2} \frac{2 \cos \theta \sin \theta}{r^{2}} \cdot \bar{\eta} \\
& \cdot\left\{j \frac{\bar{\beta}^{*}}{r}+\left(1-\frac{\bar{\beta}^{*}}{\bar{\beta}}\right) \frac{1}{r^{2}}-j \frac{1}{\bar{\beta} r^{3}}\right\} \cdot e^{-2 \alpha r}
\end{aligned}
$$

The total radiated power is obtained through integration

$$
P_{\text {total }}=\int_{0}^{2 \pi} \int_{0}^{\pi} \operatorname{Re}\left\{W_{r}\right\} \cdot r^{2} \sin \theta d \theta d \phi
$$

resulting with $\left(C_{\text {total }}=C \cdot 8 \pi / 3\right)$ :

$$
\begin{aligned}
P_{\text {total }} & =C_{\text {total }} \cdot \operatorname{Re}\{\bar{\eta}\} \cdot|\beta|^{2} \cdot e^{-2 \alpha r} \\
& +C_{\text {total }} \cdot \operatorname{Re}\left\{\bar{\eta} \cdot\left(\frac{2 \alpha}{r}+\left(1-\frac{\bar{\beta}^{*}}{\bar{\beta}}\right) \frac{1}{r^{2}}-j \frac{1}{\bar{\beta} r^{3}}\right)\right\} \cdot e^{-2 \alpha r}
\end{aligned}
$$

In this expression, a clear separation between the "far-field" and "reactive near-field" part is made. We can further use this separation idea and rearrange it to obtain the expressions for certain types of losses (losses in certain regions). Namely, using eq. (4), in the near-field the losses and corresponding efficiency $e$ can be expressed with

$$
e_{\substack{\text { losses } \\ \text { in the } \\ \text { reactive } \\ \text { near-field }}}=\frac{\exp \left(2 \alpha\left(r_{\text {far }}-r_{\text {impl }}\right)\right) \operatorname{Re}\left\{j \bar{\eta} \cdot \hat{H}_{n}^{\prime(2)}\left(\bar{\beta} r_{\text {far }}\right) \cdot \hat{H}_{n}^{(2)^{*}}\left(\bar{\beta} r_{\text {far }}\right)\right\}}{\operatorname{Re}\left\{j \bar{\eta} \cdot \hat{H}_{n}^{(2)}\left(\bar{\beta} r_{\text {impl }}\right) \cdot \hat{H}_{n}^{(2)^{*}}\left(\bar{\beta} r_{\text {impl }}\right)\right\}} .
$$

Here $r_{f a r}$ represents "large-enough" radius at which only farfield components are practically present. This expression can be approximated for the dominant spherical mode as

$$
e_{\substack{\text { losses } \\ \text { in the } \\ \text { reactive } \\ \text { near-field }}}=\frac{|\bar{\beta}|^{2} \operatorname{Re}\{\bar{\eta}\}}{\operatorname{Im}\left\{\bar{\eta} /\left(\bar{\beta} r_{\text {impl }}^{3}\right)\right\}}
$$

The propagating field absorption efficiency is equal

$$
e_{\substack{\text { propagating } \\ \text { absorption } \\ \text { losses }}}=\exp \left(-2 \alpha\left(r_{\text {body }}-r_{\text {impl }}\right)\right)
$$

and the efficiency due to reflections at the outer boundary

$$
e_{\substack{\text { losses } \\ \text { due to } \\ \text { reflections }}}=\frac{\operatorname{Re}\left\{|T|^{2} / Z_{\text {air }}\right\}}{\operatorname{Re}\left\{1 / Z_{\text {body }}\right\}}, \quad T=\frac{2 Z_{\text {air }}}{Z_{\text {air }}+Z_{\text {body }}}
$$

The mode impedance $Z$ is calculated using the equations (5) and (6). For the far-field region $Z=\eta$.

Using these expressions for different efficiencies the radiated power can be expressed as

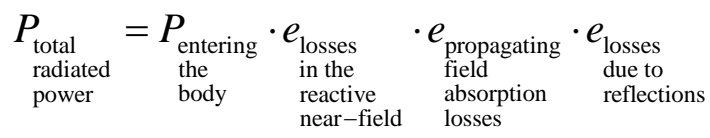

Similar can be derived for the magnetic type source case, but if we observe the expression for the total radiated power

$$
\begin{aligned}
P_{\text {total }} & =C_{\text {total }} \cdot \operatorname{Re}\left\{\frac{1}{\bar{\eta}}\right\} \cdot|\beta|^{2} \cdot e^{-2 \alpha r} \\
& +C_{\text {total }} \cdot \operatorname{Re}\left\{\frac{1}{\bar{\eta}} \cdot\left(\frac{2 \alpha}{r}+\left(1-\frac{\bar{\beta}^{*}}{\bar{\beta}}\right) \frac{1}{r^{2}}-j \frac{1}{\bar{\beta} r^{3}}\right)\right\} \cdot e^{-2 \alpha r}
\end{aligned}
$$

and by noting that the product $\bar{\beta} \bar{\eta}=\omega \mu_{0}$ is a real number, we can simplify the upper expression as

$$
P_{\text {total }}=C_{\text {total }} \cdot \operatorname{Re}\left\{\frac{1}{\bar{\eta}}\right\} \cdot|\beta|^{2} \cdot e^{-2 \alpha r}+C_{\text {total }} \cdot \operatorname{Re}\left\{\frac{1}{\bar{\eta}} \cdot \frac{2 \alpha}{r}\right\} \cdot e^{-2 \alpha r}
$$

This is additional (mathematical) explanation why the magnetic dipole has much less absorbed power in the near field (since there are no high-order terms in the expression for the total radiated power). Therefore, the near-field losses and the corresponding efficiency for the implanted magnetic dipole can be expressed as

$$
e_{\substack{\text { losses } \\ \text { in the } \\ \text { reactive } \\ \text { near-field }}}=\frac{\exp \left(2 \alpha\left(r_{\text {far }}-r_{\text {impl }}\right)\right) \operatorname{Re}\left\{-j \bar{\eta} \cdot \hat{H}_{n}^{(2)}\left(\bar{\beta} r_{\text {far }}\right) \cdot \hat{H}_{n}^{(2)^{*}}\left(\bar{\beta} r_{\text {far }}\right)\right\}}{\operatorname{Re}\left\{-j \bar{\eta} \cdot \hat{H}_{n}^{(2)}\left(\bar{\beta} r_{\text {impl }}\right) \cdot \hat{H}_{n}^{\prime(2)^{*}}\left(\bar{\beta} r_{\text {impl }}\right)\right\}},
$$

or approximately 


$$
e_{\substack{\text { losses } \\ \text { reactive } \\ \text { near-field } \\ \text { near-e }}}=\frac{|\bar{\beta}|^{2}}{2 \alpha / r_{\text {impl }}}
$$

A quick calculation for our test case gives

TABLE I

TOTAL RADIATED POWER REACHING FREE SPACE.

\begin{tabular}{|l|c|c|}
\hline & $\begin{array}{c}\text { Electric } \\
\text { dipole }\end{array}$ & $\begin{array}{c}\text { Magnetic } \\
\text { dipole }\end{array}$ \\
\hline$P_{\text {entering the body }(\mathrm{dBm})}$ & 0 & 0 \\
\hline$e_{\text {losses in the reactive near-field }}(\mathrm{dB})$ & -34.2 & -10.2 \\
\hline$e_{\text {propagating field absorption losses }(\mathrm{dB})}$ & -16.1 & -16.1 \\
\hline$e_{\text {losses due to reflections }}(\mathrm{dB})$ & -6.2 & -7.5 \\
\hline $\begin{array}{l}\text { Total radiated power }(\mathrm{dB})- \\
\text { calculated using }(17)\end{array}$ & -56.5 & -33.8 \\
\hline $\begin{array}{l}\text { Total radiated power }(\mathrm{dBm})- \\
\text { calculated rigorously }\end{array}$ & -56.4 & -33.7 \\
\hline
\end{tabular}

The results shown so far focused on describing the loss mechanisms in the body and they were demonstrated using sources placed in the center of the sphere. A more practical situation would be to have the sources placed closer to the body - air boundary and one would expect that due to loss of symmetry the loss mechanisms will be affected. However, Fig. 7 shows the case when the distance of the source from the body - air interface is fixed and the radius of the body is changed, and we see that the behavior is the same as in the center case. There is an additional drop of the total radiated power (since the body is larger in that case), but this change can be predicted using diagrams in Fig. 7.

The other term of interest would be the maximum power density that we can get just outside the body (where we could put on-body antenna to establish the communication link). If we fix the distance of the antenna to the body interface, we can see that the same efficiency expressions can be used also for this scenario (with the assumption that the orientation of the dipole is transverse to direction of observation). Fig. 8 shows the radial component of the power density evaluated at different points on the radial line connecting the center of the body and the feed point (i.e. on the $x$-axis with $x>r_{\text {feed }}$ ). Here the position of the implanted antenna is kept fixed at $9 \mathrm{~cm}$ distance from the body boundary, and the radius of the spherical body is taken as a parameter. Note that the power density is normalized with the factor $W_{0} /(8 \pi / 3) \cdot r^{2}=W_{0} /(8 \pi / 3) \cdot\left(r_{\text {obs }}-r_{\text {feed }}\right)^{2}$, were $W_{0}$ is the maximum value of the real part of the power density component normal to the surface of implanted antenna, i.e., just inside the lossy medium.

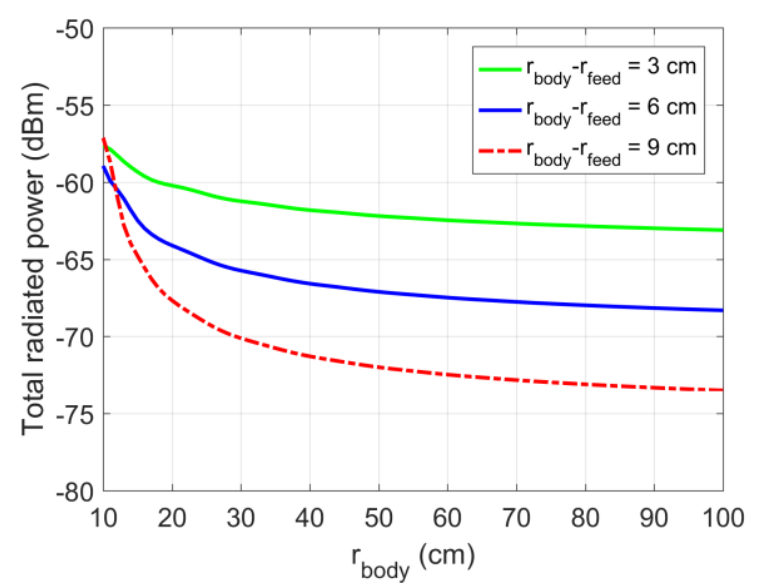

(a)

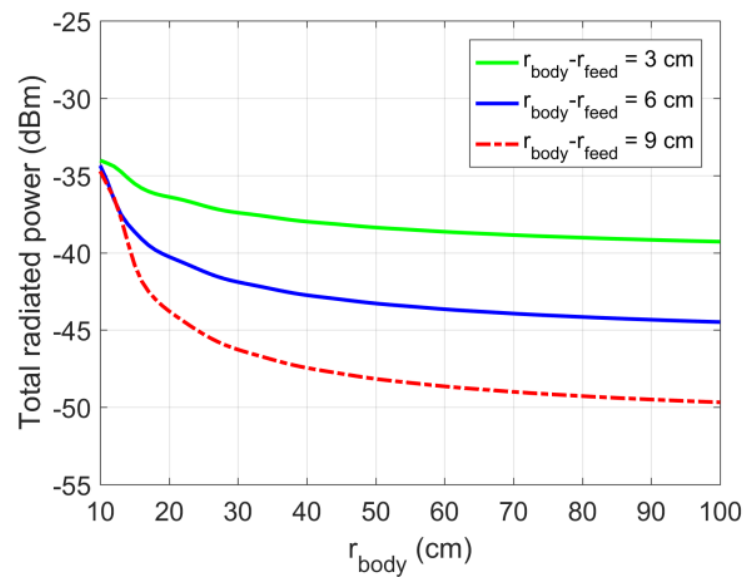

(b)

Fig. 7. Total radiated power as a function of body radius when the source is kept fixed at a certain distance from the boundary; (a) electric dipole case, (b) magnetic dipole case

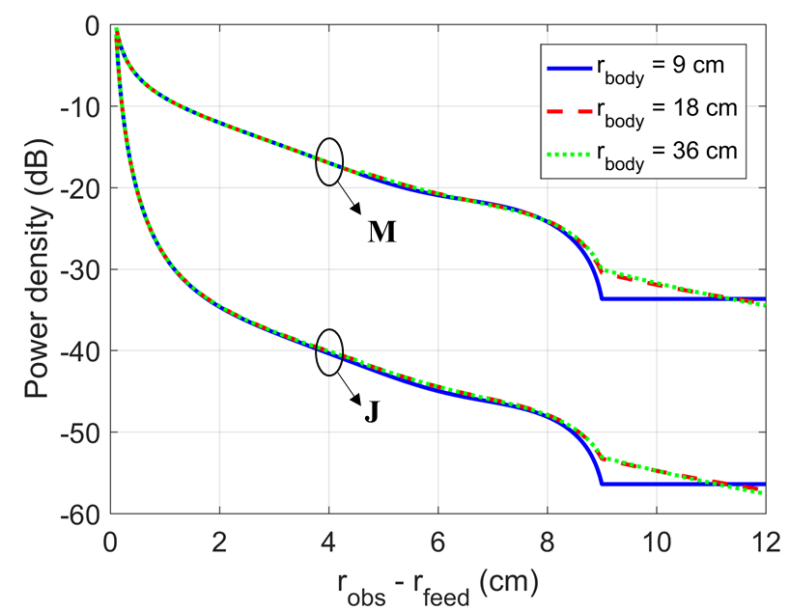

Fig. 8. Radial component of the normalized power density at different distances from the implanted antenna. The source is kept fixed at $9 \mathrm{~cm}$ distance from the body boundary. Results for the electric and magnetic dipole are shown. 


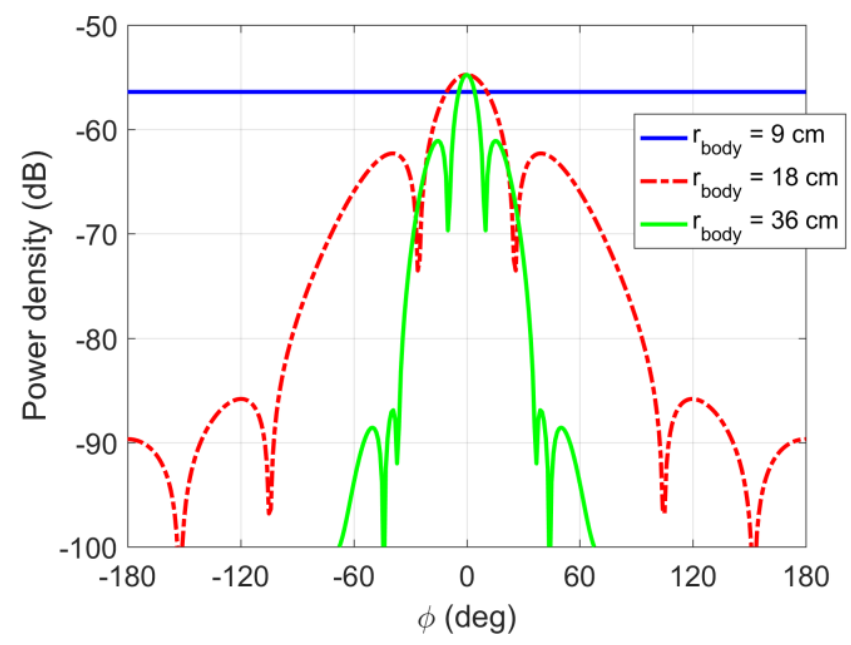

Fig. 9. Angular dependency of the normalized power density as a function of dielectric sphere radius. The $z$-oriented electric dipole is located at the $x$-axis 9 $\mathrm{cm}$ away from the boundary in all cases (Fig. 1) and the angular dependency is taken in $x y$ plane $1 \mathrm{~cm}$ away from the outside boundary of the dielectric sphere.

The focusing effect of the dielectric body is illustrated in Fig. 9 in which the angular dependency of the normalized power density is shown as a function of dielectric sphere radius. Like in the previous case the source is kept fixed at $9 \mathrm{~cm}$ distance from the body boundary and the power density is calculated at $1 \mathrm{~cm}$ distance from the outside boundary of the dielectric sphere. It can be seen that value of the power density strongly depends on the distance from the source, i.e. on the amount of propagating field absorption losses. In other words, for the maximum value of the power density outside the body the shape and dimension of the host medium has very little importance.

Further investigation of this scenario is shown in Fig. 10 which shows that the maximum of power density just outside the body mostly depends on the distance from the implanted antenna, i.e. that in principle does not depend on the body radius. In the concentric case (when the implanted antenna is placed in the origin of the body) there is only stronger influence of the efficiency due to reflections at the outer boundary. However, when this symmetry is broken, the influence of reflection is much weaker. This leads to a conclusion that the assumptions and approximate expressions derived earlier can be also used for practical implant cases when they are located relatively close to the body - air boundary.

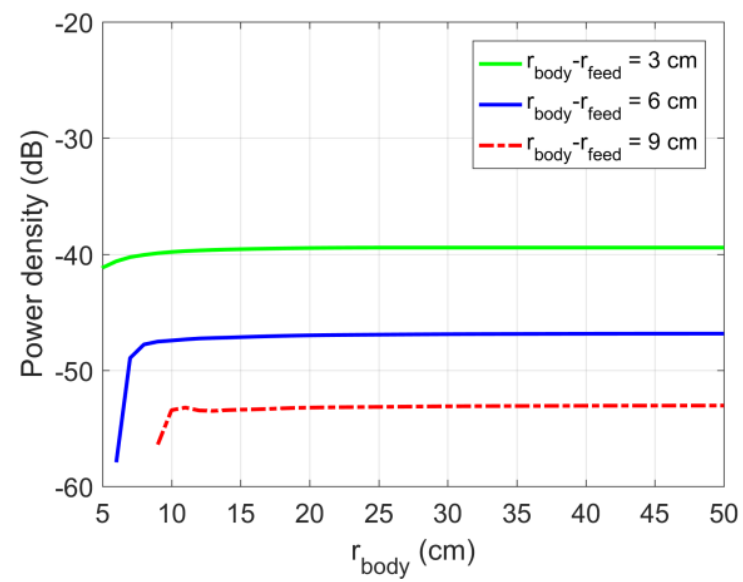

(a)

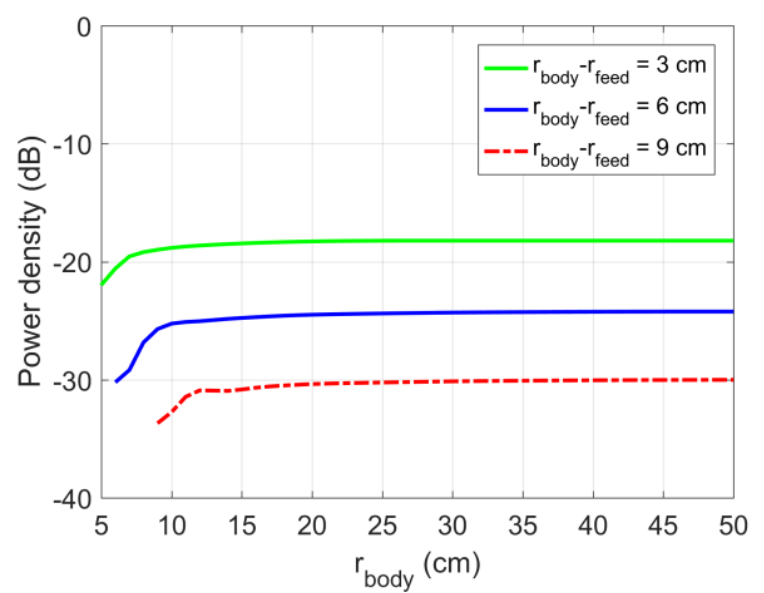

(b)

Fig. 10. Power density as a function of the body radius when the source is kept fixed at a certain distance from the boundary; (a) electric dipole, (b) magnetic dipole.

Therefore, we can conclude that the maximum power density that is obtainable from the implantable antenna located in a body of arbitrary shape and dimensions is equal:

(a) for the magnetic type of antenna of radius $r_{i m p l}$ placed at distance $\Delta$ inside the body

$$
W_{\max }=W_{0} \frac{r_{i m p l}^{2}}{\Delta^{2}} \frac{|\bar{\beta}|^{2}}{2 \alpha / r_{i m p l}} \exp \left(-2 \alpha\left(\Delta-r_{i m p l}\right)\right)
$$

(b) for the electric type of antenna of radius $r_{\text {impl }}$ placed at distance $\Delta$ inside the body

$$
W_{\max }=W_{0} \frac{r_{i m p l}^{2}}{\Delta^{2}} \frac{|\bar{\beta}|^{2} \operatorname{Re}\{\bar{\eta}\}}{\operatorname{Im}\left\{\bar{\eta} /\left(\bar{\beta} r_{i m p l}^{3}\right)\right\}} \exp \left(-2 \alpha\left(\Delta-r_{\text {impl }}\right)\right) \text {. }
$$

Note that in our spherical model $\Delta=r_{\text {body }}-r_{\text {feed }}$. Furthermore, the maximum bound does not take into account losses due to reflections since these losses depend on the body boundary properties. A good approximation can be obtained with the large-radius variation of eq. (16): 


$$
e_{\begin{array}{c}
\text { losses } \\
\text { due to } \\
\text { reflections }
\end{array}} \approx\left|\frac{2 \sqrt{\varepsilon_{\text {body }}}}{1+\sqrt{\varepsilon_{\text {body }}}}\right|^{2} / \operatorname{Re}\left\{\sqrt{\varepsilon_{\text {body }}}\right\} .
$$

In order to illustrate the limit for maximum power density obtainable from the implanted antenna two design diagrams containing power density limits are shown in Fig. 11. In the first case the working frequency $(f=403.5 \mathrm{MHz})$ and the type of tissue (muscle tissue with $\varepsilon_{\mathrm{r}}=57.1-j 35.51$ ) are fixed. As a parameter we took the radius of the implanted antenna and the position (depth) of the antenna inside the body. We considered the electric type of implanted antenna. The diagram gives the relation between the affordable losses in the body, position of the antenna inside the body and the size of the capsule. Also plotted as a small triangle in Fig. 11.a is the estimated power density for the antenna described in [23] (the capsule in [23] has a shape of a pill with dimensions $17 \times ø 7 \mathrm{~mm}$ and it is evaluated in spherical phantom with $50 \mathrm{~mm}$ radius). Here for the dimension of the equivalent spherical implanted antenna we took $\varnothing 10 \mathrm{~mm}$ (defined with the size of the antenna inside the capsule) and since the estimated power density obtained in [23] is $W / W_{0}=-24.2 \mathrm{~dB}$, which includes losses due to reflection, we can conclude that the radiation properties of this antenna are near the limit. In Fig. 11.b we illustrated the influence of different types of tissues on the amount of losses inside the body. The working frequency is again $f=403.5 \mathrm{MHz}$, the radius of the implanted antenna is $r_{\text {impl }}=4 \mathrm{~mm}$ and the position (depth) of the implanted antenna inside the body is $\Delta=90 \mathrm{~mm}$. Between the considered tissues, bones introduce the smallest losses, while the largest losses are due to muscle and brain tissues.

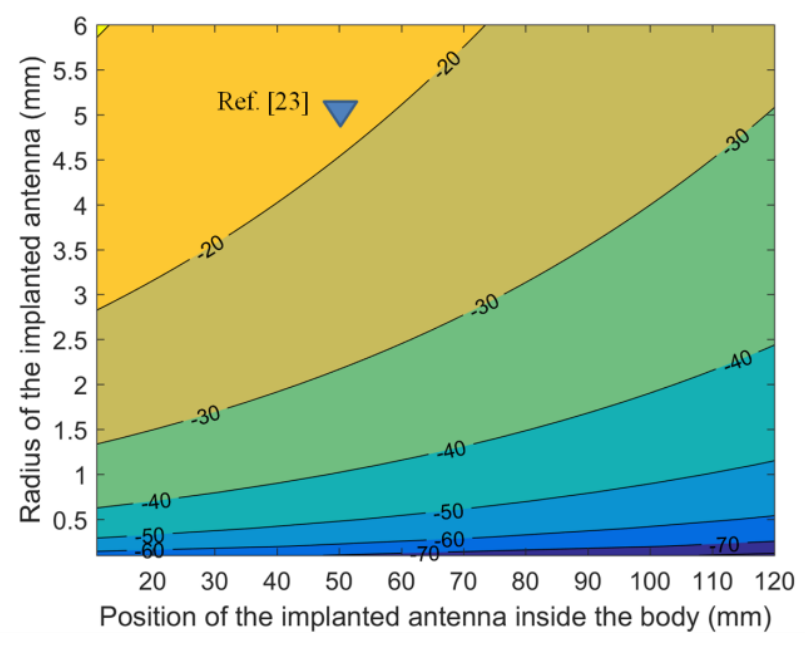

(a)

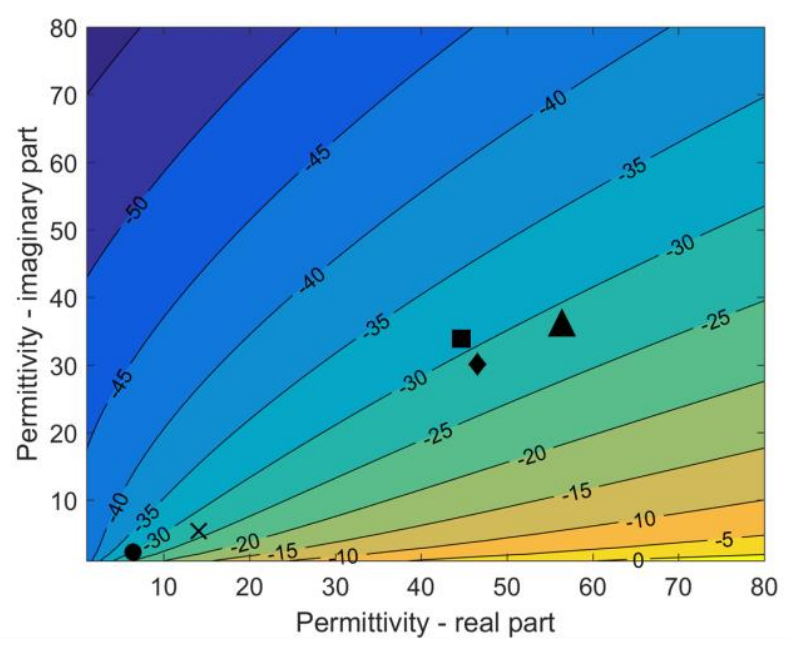

(b)

Fig. 11. Maximum power density $W / W_{0}(\mathrm{~dB})$ obtainable from a implanted antenna of electric type; (a) as a function of size and position of implanted antenna, and (b) as a function of complex permittivity $\left(\boldsymbol{\Delta}-\right.$ muscle tissue $\varepsilon_{r}=$ $57.1-j 35.51, \bullet-$ fat tissue $\varepsilon_{r}=5.58-j 1.8,-$ dry skin $\varepsilon_{r}=46.7-\mathrm{j} 30.72$, $\mathbf{X}$ - bone $\varepsilon_{r}=13.1-j 4.0, \mathbf{-}$-brain - IEEE head model $\left.\varepsilon_{\mathrm{r}}=43.50-j 34.75\right)$.

\section{CONCLUSION}

The knowledge of fundamental radiation limits for antennas implanted in a lossy medium is instrumental in the design of such implants. These limits were studied assuming elementary radiating sources, a spherical model for the host body and a spherical expansion to describe the electromagnetic fields inside the structures. With this model, first the loss mechanisms were analyzed and it was shown the losses can be divided into three contributions; (i) close to the implant, the losses due to the coupling of the near field and the lossy biological host tissue, (ii) the losses due to the field propagating through the body, (iii) the reflection at the body free space interface. The last two loss contributions are unavoidable for the signal to reach free space, however, the antenna designer should work on the first to minimize the loss.

Based on this separation the upper bound was obtained for the power density that can reach free space from an implant, depending only on the frequency, the dielectric permittivity of the biological tissue, the depth of the implant and the size of the implant encapsulation. This is vital information for comparing potential antennas and also gives a maximal bound for the link budget between an implant and an on body antenna.

Finally, as demonstrated, the critical dimension for this upper bound is the depth of the implant, not the shape of the phantom. Thus, the results are quite universal and directly usable in practice for host bodies of other shapes. 


\section{ACKNOWLEDGMENT}

This work has been supported in part by Croatian Science Foundation under the project IP-11-2013-3425.

\section{REFERENCES}

[1] C.A. Balanis, Antenna Theory, Analysis and Design, Harper and Row, 1982, New York

[2] L.J. Chu, "Physical limitations on omni-directional antennas", Journal of Applied Physics,. 19, 1948, pp. 1163-1175.

[3] H.A. Wheeler, "Fundamental Limitations of Small Antennas", Proc. of the IRE, 1947, pp. 1479-1484.

[4] R.F. Harrington, "On the Gain and Beamwidth of Directional Antennas", IRE Transactions on Antennas and Propagation, vol. AP-6, 1958, pp. 219-225.

[5] R.E Collin and S. Rothschild, "Evaluation of Antenna Q", IEEE Transactions on Antennas and Propagation, vol. AP-12, 1964, pp. 2327.

[6] R.L. Fante, "Quality factor of general ideal antennas", IEEE Transactions on Antennas and Propagation, vol. AP-17, 1969, pp. 151155.

[7] Fano, R. M., "Theoretical limitations on the broadband matching of arbitrary impedances", J. Franklin Inst. 249, 57-83 see also 139-154, 1950.

[8] A.D. Yaghjian and S.T. Best, "Impedance, Bandwidth and Q of Antennas", IEEE Transactions on Antennas and Propagation, vol. AP53, 2005, pp. 1298-1324.

[9] J.S. McLean, "A Re-Examination of the Fundamental Limits on the Radiation Q of Electrically Small Antennas", IEEE Transactions on Antennas and Propagation, vol. AP-44, 1996, pp. 672-676.

[10] R.C. Hansen, Electrically small, Superdirective and Superconductive Antennas, John Wiley and sons, New Jersey, 2006.

[11] H.D. Foltz and J.S. McLean, "Limits on the Radiation Q of Electrically Small Antennas Restricted to Oblong Bounding Regions", Proceedings of the IEEE Antennas and Propagation Symposium, 1999, pp. 27022705.

[12] J.C.E. Sten, P.K. Koivisto, A. Hujanen, "Limitations for the Radiation Q of a Small Antenna Enclosed in a Spheroidal Volume: Axial Polarization", Int. Journal Electron. Communi. (AË̈), vol. 55, 2001, pp. 198-204.

[13] J.C.E. Sten, "Radiation Q of a Small Antenna Enclosed in an Oblate Spheroidal Volume: Transverse to Axis Polarization", Int. Journal Electron. Communi. (AEÜ), vol. 57, 2003, pp. 201-205.

[14] S.R. Best and A.D. Yaghjian, "The Lower Bound on Q for Lossy Electric and Magnetic Dipole Antennas", IEEE Antennas and Wireless Propagation Letters, vol. 3, 2004, pp. 314-316.

[15] A.D. Yaghjian, "Improved Formulas for the Q of Antennas with Highly Lossy Dispersive Materials", IEEE Antennas and Wireless Propagation Letters, vol. 5, 2006, pp. 365-369.

[16] M Gustafsson, C. Sohl and G. Kristensson, "Physical limitations on antennas of arbitrary shape", Proc. R. Soc. A, vol. 463, pp. 2589-2607, 2007.

[17] M. Gustafsson, C. Sohl and G. Kristensson, "Illustrations of New Physical Bounds on Linearly Polarized Antennas", IEEE Transactions on Antennas and Propagation,, Vol. AP-57, No. 5, pp. 1319-1327, 2009.

[18] C. Ehrenborg and M. Gustafsson, "Calculating physical bounds for inbody antennas", Proc. the $12^{\text {th }}$ European Conference on Antennas and Propagation, EuCAP 2018, London, UK, pp 1-3.

[19] A.K. Skrivervik, J.-F. Zürcher, O. Staub and J.R. Mosig, " PCS Antenna Design : The Challenge of Miniaturization", IEEE AP-Magazine, Aug. 2001, pp12-27

[20] N. H. M. Rais, P. J. Soh, F. Malek, S. Ahmad, N.B.M. Hashim, P.S Hall, "A review of wearable antenna", 2009 Loughborough Antennas \& Propagation Conference, Loughbourough, UK, pp. 225-228

[21] B. Gupta, S. Sankaralingam, S. Dhar, "Development of wearable and implantable antennas in the last decade: A review", Mediterranean Microwave Symposium, Cyprus, 25-27 Aug. 2010, pp. 251-267.

[22] A. Pellegrini, A. Brizzi, L. Zhang, K. Ali, Y. Hao, X. Wu, C. C. Constantinou, Y. Nechayev, P. S. Hall, N. Chahat, M. Zhadobov, R. Sauleau," Antennas and Propagation for Body-Centric Wireless
Communications at Millimeter-Wave Frequencies: A Review", IEEE antennas and propagation magazine, vol. 55, issue 4, pp. 262-287, 2013.

[23] D. Nikolayev, M. Zhadobov, L. Le Coq, P. Karban and R, Sauleau, "Robust ultraminiature capsule antenna for ingestible and implantable applications", ", IEEE Transactions on Antennas and Propagation,, vol. AP-65, No. 11, pp. 6107-6119, 2017.

[24] J. Faerber et al., "In vivo characterization of wireless telemetry module for a capsule endoscopy system utilizing a conformal antenna," IEEE Transaction on Biomedical Circuits and Systems, vol. 12, pp. 95-104, Feb. 2018.

[25] R. Moore, "Effects of a surrounding conducting medium on antenna analysis," IEEE Transactions on Antennas and Propagation, vol. AP11, no. 3, pp. 216-225, May 1963.

[26] A. Sommerfeld, Partial differential equations in physics, Academic Press, New York, 1949.

[27] F. Merli, L. Bolomey, J.-F. Zürcher, G. Corradini, E. Meurville and A.K. Skrivervik, "Design, realization and measurements of a miniature antenna for implantable wireless communication systems", IEEE Transactions on Antennas and Propagation, vol. AP-59, issue 10, 2011, pp. $2544-2555$

[28] F. Merli, B. Fuchs, J.R. Mosig and A.K. Skrivervik, "The effect of insulating layers on the performance of implanted antennas", IEEE Transactions on Antennas and Propagation, vol. AP-59, Nr. 1, pp. 2131, 2011.

[29] A.K. Skrivervik, Implantable antennas: The challenge of efficiency, Proc. the $7^{\text {th }}$ European Conference on Antennas and Propagation, EuCAP 2013, Gothenburg, Sweden, pp. 1-5.

[30] J. Kim and Y. Rahmat-Samii, "Implanted antenna inside a human body: simulations, design, and characterization," IEEE Transactions on Antennas and Propagation, vol. AP-52, 2004, pp. 1934-1943.

[31] M. Bosiljevac, Z. Sipus, A.K. Skrivervik, "Propagation in Finite Lossy Media: an Application to WBAN", IEEE Antennas and Wireless Propagation Letters, vol.14, pp. 1546 - 1549, 2015.

[32] M. Bosiljevac, Benjamin Fuchs, A.K. Skrivervik and Z. Sipus, "Study of wearable WBAN antenna properties based on spherical body model", Proc. the $10^{\text {th }}$ European Conference on Antennas and Propagation, EuCAP 2013, Davos, Switzerland, pp 1-3.

[33] Evaluating Compliance With FCC Guidelines for Human Exposure to Radiofrequency Electromagnetic Fields, 97-01 ed. Washington, DC: Federal Communication Commission (FCC) Std. Supplement C, OET Bulletin 65, p. 35, 2001.

[34] J. A. Stratton, Electromagnetic theory, McGraw-Hill N.Y.,1941.

[35] R. F. Harrington, Time Harmonic Electromagnetic Fields, McGraw-Hill, New York, 1961

[36] W.C. Chew, Waves and fields in inhomogeneous media, IEEE Press, N.Y., 1995.

[37] Z. Sipus, P.-S. Kildal, R. Leijon, and M. Johansson, "An Algorithm for Calculating Green's Functions for Planar, Circular Cylindrical and Spherical Multilayer Substrates," Applied Computational Electromagnetics Society Journal, Vol. 13, pp. 243-254, Nov. 1998.

[38] V.B.G. Truong, M. Thiel, A. Dreher, "A unified approach to the analysis of radial waveguides, dielectric resonators, and microstrip antennas on spherical multilayer structures, IEEE Trans. Microwave Theory Tech., vol. 53, pp. 404 - 409, Jan. 2005.

[39] O. R. Cruzan, "Translational Addition Theorems for Spherical Vector Wave Functions", Quart. Appl. Math., Vol. 20, pp. 33-40, 1962.

[40] S. Stein, "Addition Theorems for Spherical Wave Functions", Quart. Appl. Math. Vol. 19, pp. 15-24, 1961.

[41] J.H. Bruning and Y.T. Lo, "Multiple Scattering of EM Waves by Spheres Part I - Multiple Expansion and Ray Optical Solutions, IEEE Trans. Antennas Propag., vol. AP-19, pp. 378-390, May 1971.

[42] M. Abramowitz and I. A. Stegun: Handbook of Mathematical Functions, Dover Books on Advanced Mathematics, Dover, New York, 1965. 


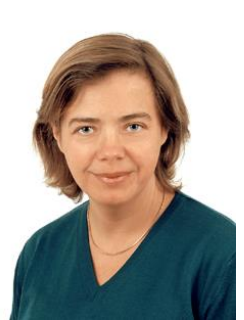

Anja Skrivervik obtained her electrical engineering degree from Ecole Polytechnique Fédérale de Lausanne in 1986, and her $\mathrm{PhD}$ from the same institution in 1992, for which she received the Latsis award. After a stay at the University of Rennes as an invited Research Fellow and two years in the industry, she returned part time to EPFL as an Assistant Professor in 1996, and is now a Professeur Titulaire at this institution, where she is the head of the Microwave and Antenna Group. Her research activities include electrically small antennas, antennas in biological media, multifrequency and ultra-wideband antennas, and numerical techniques for electromagnetics. She is author or co-author of more than 200 peer reviewed scientific publications. Her teaching activities include courses on microwaves and antennas, and she has course at Bachelor, Master and PhD levels. She was director of the EE section form 1996-2000.

She is very active in European collaboration and European projects. She was the chairperson of the Swiss URSI until 2012, is a Board member of the European School on Antennas and is frequently requested to review research programs and centers in Europe. She is a member of the board of directors of the European Association on Antennas and Propagation (EurAAP) since 2017.

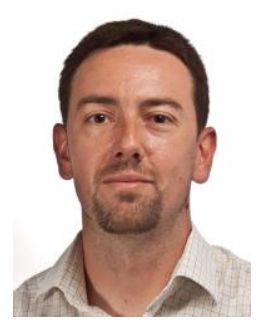

Marko Bosiljevac received B.Sc., and Ph.D. degrees in electrical engineering from the University of Zagreb, Faculty of Electrical Engineering and Computing (FER), Zagreb, Croatia, in 2005 and 2012, respectively. From March 2006 he is working at the Department of Wireless Communications at FER. He was a visiting researcher at the University of Siena, Italy in total for 8 months between October 2010 and March 2012. In March 2016 he was promoted to Assistant Professor at the Department of Wireless Communications at FER. He published more than 40 papers in journals and conference proceedings in the area analytical and numerical modelling and development of various electromagnetic and optical structures and systems. He also participated in the organization of several doctoral schools and international conferences and he serves as a technical reviewer for several international scientific journals. He received silver medal "Josip Lončar" from FER for outstanding Ph.D. thesis in 2012.

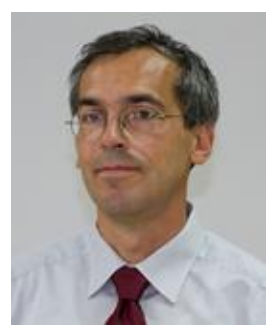

Zvonimir Sipus received the B.Sc. and M.Sc.degrees in electrical engineering from the University of Zagreb, Zagreb, in 1988 and 1991, respectively, and the Ph.D. degree in electrical engineering from the Chalmers University of Technology, Gothenburg, Sweden, in 1997.

From 1988 to 1994, he was with the Rudjer Boskovic Institute, Zagreb, as a Research Assistant, where he was involved in the development of detectors for explosive gasses. In 1994, he joined the Antenna Group, Chalmers University of Technology, where he was involved in research projects concerning conformal antennas and soft and hard surfaces. In 1997, he joined the Faculty of Electrical Engineering and Computing, University of Zagreb, where he is currently a Professor. From 1999 to 2005, he was also an Adjunct Researcher with the Department of Electromagnetics, Chalmers University of Technology. Since 2006, he has been involved in teaching with the European School of Antennas. From 2008 to 2012 and from 2014 to 2018, he has been the Head of the Department of Wireless Communications. His current research interests include the analysis and design of electromagnetic structures with application to antennas, microwaves, and optical communication and sensor systems. 